\title{
Modelamiento del ciclo de la construcción en Colombia mediante dinámica de sistemas ${ }^{*}$
}

\author{
Miguel David Rojas López* \\ Carlos Roberto Arango*** \\ Lina Bastidas ${ }^{* * *}$
}

Recibido: 04/09/2015 - Aceptado: 02/05/2016

DOI: $10.22395 /$ rium.v15n29a3

\begin{abstract}
Resumen
El objetivo de esta investigación es proponer un modelo mediante dinámica de sistemas de los determinantes del sector de la construcción en Colombia, admitiendo que el comportamiento es cíclico; es decir, si para el año 2015 el sector se encuentra creciendo debido a la cantidad de obras que se están ejecutando en infraestructura, como la Cuarta Generación de Concesiones y los planes de vivienda del Gobierno, en otros momentos (como en el 2012) la construcción decreció.
\end{abstract}

Palabras clave: vivienda, dinámica de sistemas, crecimiento económico.

Artículo resultado de proyecto de investigación asociado a una tesis de maestría en ingeniería administrativa en la Facultad de Minas de la Universidad Nacional de Colombia. El proyecto de investigación se ejecutó con recursos propios del segundo autor y con el apoyo del grupo de Investigación CINCO de la Universidad Nacional de Colombia.

* Ph. D. Ingeniería, profesor asociado, director Centro de Investigación y Consultoría Organizacional (CINCO), Departamento de Ingeniería de la Organización, Facultad de Minas, Universidad Nacional de Colombia, Sede Medellín. Carrera 80 N. ${ }^{\circ}$ 65-223 Bloque M8B oficina 202 Medellín, Tel 4255225-4255309. mdrojas@unal.edu.co

** Magíster en Ingeniería Administrativa, Ingeniero Civil, Centro de Investigación y Consultoría Organizacional (CINCO), Departamento de Ingeniería de la Organización, Facultad de Minas, Universidad Nacional de Colombia, Sede Medellín. Correo electrónico crarangog@unal.edu.co

*** Magíster en Sistemas, ingeniera administrativa Centro de Investigación y Consultoría Organizacional (CINCO). Correo electrónico: 1mbastid@unal.edu.co 


\section{Modeling construction cycle in Colombia by system dynamics}

\section{Abstract}

The objective of this investigation is to propose a model using dynamic systems about determinants of the construction sector in Colombia assuming that such behavior is cyclical. The indicators used to track the cyclical behavior includes: interest rate for housing loans, number of building permits requested, number of houses built and number of credits approved by the banking system, among others. From these data and statistical analysis, the housing deficit and the growth in the share of construction activity in the gross domestic product (gdp) of Colombia is quantified.

Key words: housing construction, dynamic systems, and economic growth. 


\section{INTRODUCCIÓN}

El sector de la construcción en Colombia presenta un comportamiento cíclico, evidenciado en indicadores recopilados por el Departamento Nacional de Estadística -DANE-, el Departamento Nacional de Planeación -DNP- y la Cámara Colombiana de la Construcción -CAMACOL-, organizaciones que analizan el comportamiento sectorial [1].

El Gobierno nacional y el sector privado reconocen la existencia del comportamiento cíclico en la actividad constructora. En algunos casos es posible diferenciar en qué punto se encuentra la actividad, es decir, si existe crecimiento o decrecimiento económico según la variación puntual de algún indicador sectorial. Se tienen herramientas para predecir el comportamiento en el mediano plazo, y teniendo en cuenta las políticas del Gobierno de turno en función de indicadores empleados, se analiza el futuro del sector.

Algunos ejemplos de cambios externos son: los subsidios a la tasa de crédito hipotecario, los planes de inversión en construcción de vivienda y los replanteos normativos asociados al licenciamiento que marcan tendencias y representatividad en el sector.

\section{MODELOS TEÓRICOS}

La modelación de interacciones de las variables que inciden en el fenómeno de la construcción de vivienda en Colombia fue presentada por Saldarriaga [2] como resultado de planteamientos de interacciones expuestas por diferentes autores desde finales de los años 90 y comienzos de la década del 2000. Saldarriaga partió del modelo publicado por Throop en el artículo "Financial Deregulation, Interest Rates and the Housing Cycle" [3] para el mercado americano, así como en las aproximaciones de Topel y Rosen en "Housing Investment in the United States" [4]. El estudio incluyó los trabajos de Cárdenas y Bernal [5], proponiendo un modelo de oferta y demanda para identificar los determinantes de la actividad en una regresión econométrica. Cárdenas et al. [6] presentaron nuevas aproximaciones al modelo con las mismas apreciaciones.

Otros autores presentan por separado una ecuación de oferta y una de demanda para el fenómeno [7], así como un modelo de ecuaciones simultáneas por el método de máxima verosimilitud con información completa. En el estudio concluyen que el precio de vivienda tiene un efecto negativo en la demanda, al igual que el desempleo y la tasa de crédito hipotecaria.

CAMACOL [8] presenta referencias al mismo proceso investigativo, y también correlaciones bivariadas con datos del 2007. Esta metodología también fue presentada por Chirivi y Pulido C [9]. 
Este último informe presenta el uso del suelo como una de las variables determinantes del ciclo de construcción, ya que los nuevos proyectos requieren la modificación de los Planes de Ordenamiento Territorial-POT-y la adecuación de terrenos para incrementar el costo de las viviendas proyectadas, alterando la ecuación de oferta planteada.

\section{DETERMINANTES DEL CICLO DE LA CONSTRUCCIÓN}

\subsection{El PIB de edificaciones}

El PIB de edificaciones es la fuente de la medición del comportamiento de la construcción en Colombia, y sirve para establecer la eficiencia de las políticas gubernamentales aplicadas al sector [10]. Es elaborado con información obtenida del DANE.

Para el DANE, las mediciones estadísticas del sector construcción las componen los subsectores de edificaciones y de obras civiles, a los cuales aplican metodologías diferentes según las particularidades de plazo, inversión, monto y estructura de proyectos. Los dos subsectores tienen, respectivamente, participaciones promedio del 2,74 \% y 2,85\% del PIB total nacional desde el año 2000, como se muestra en la figura 1. El subsector de edificaciones está relacionado con la construcción o reparación de inmuebles y con la actividad de oferta y demanda de vivienda. El segundo subsector de obras civiles contempla el comportamiento sectorial en la construcción o reparación de obras de infraestructura vial, eléctrica, aeroportuaria, entre otras, que corresponden a la medición de la inversión y desarrollo de la infraestructura.

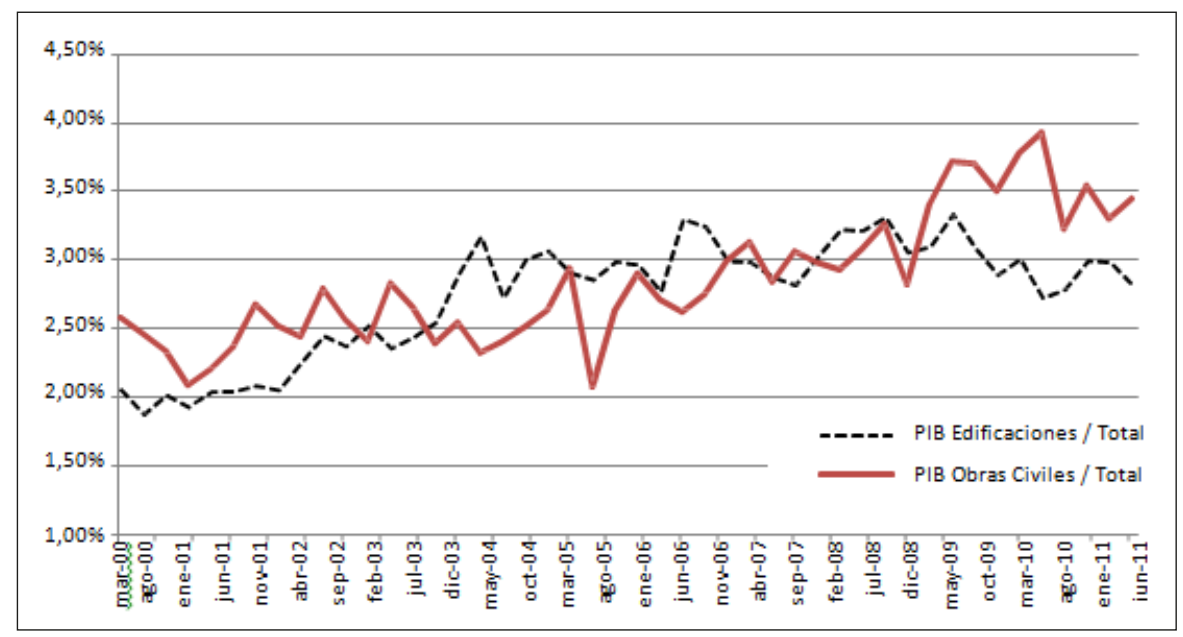

Figura 1. PIB de edificaciones y obras civiles en el PIB total Fuente: [10] 
La figura 1 muestra que el PIB de obras civiles es mayor que el PIB de edificaciones para enero de 2011, y ambos muestran una tendencia creciente.

El subsector edificación incluye: residencial, no residencial, y reparaciones y servicios. El PIB de edificaciones mide el valor agregado que genera el valor bruto de la producción, es decir, $\mathrm{m}^{2}$ construidos por precios comerciales, menos el costo directo de construcción (ver figura 2). En la última década la participación del PIB de edificaciones en el PIB total oscila entre el 1,8 \% y el 3,3\%.

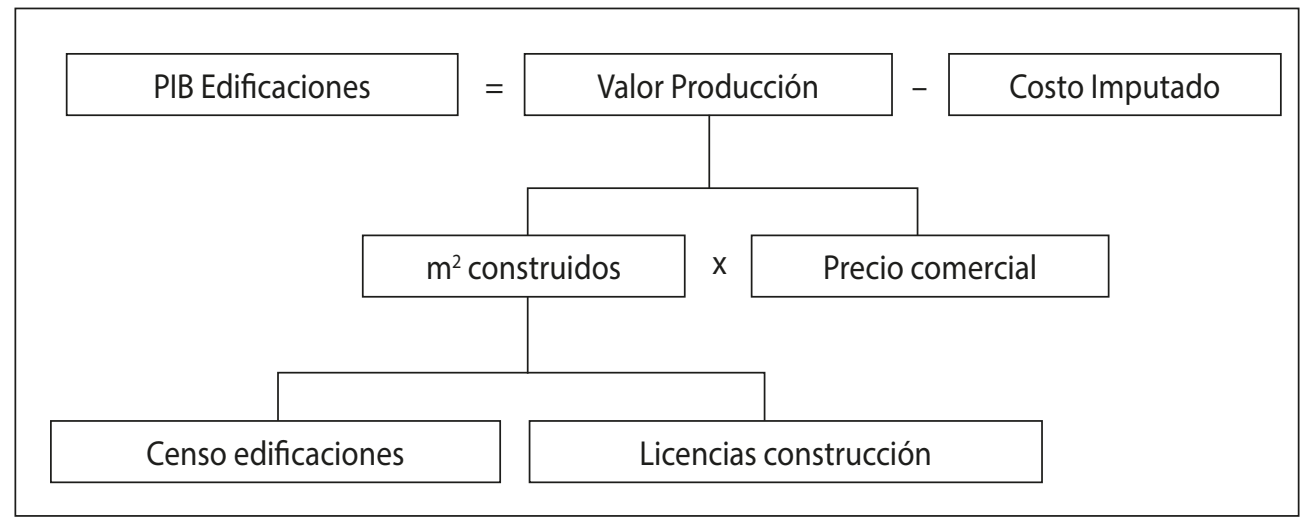

Figura 2. Determinación del PIB de edificaciones

Fuente: [10]

Los $\mathrm{m}^{2}$ construidos corresponden a la variable integrada en la medición del DANE, diferente de $\operatorname{los} \mathrm{m}^{2}$ licenciados, involucrando el principio de causación que mide el efecto en las etapas del proceso constructivo sobre el PIB, información obtenida a partir de los resultados del censo trimestral de edificaciones-CEED-, proveniente de 15 áreas urbanas, y de estadísticas de licenciamiento -ELIC-, encargadas de estimar participaciones de zonas no cubiertas por el censo.

Los coeficientes de incidencia que establece la metodología del DANE permiten asignar a $\operatorname{los} \mathrm{m}^{2}$ construidos del período, el porcentaje de costo que refleja cada etapa constructiva en los diferentes tipos de construcciones involucradas en el censo. Para el año 2009 y 2010, el indicador recopilado con la información del DANE presentó inconsistencias en el comportamiento del sector real, medido desde la percepción de CAMACOL [10]; mientras los estímulos a créditos de vivienda otorgados por el sistema bancario, los subsidios por parte del Gobierno y la actividad a lo largo de la cadena de valor aumentaban, el PIB edificador registraba la mayor caída de la década. Esto motivó un proceso de entendimiento del fenómeno por parte de ambos estamentos con el fin de entender las relaciones de causalidad y temporalidad de los indicadores y el PIB sectorial. El análisis de las desviaciones observadas en el período 2009-2010 
indica cambios metodológicos en la valoración estadística de los $\mathrm{m}^{2}$ construidos, más que un problema de validación de la actividad sectorial con el indicador empleado tradicionalmente.

La fuente de valor agregado del sector valorado en el PIB corresponde a las edificaciones no residenciales, incluyendo las destinadas a comercio y hotelería. Según CAMACOL el $75 \%$ del total de los $\mathrm{m}^{2}$ construidos corresponde a vivienda, aunque el resultado del aporte de este sector al PIB de edificaciones corresponde al $48 \%[10,8]$.

Las conclusiones del análisis elaborado entre CAMACOL y el DANE, muestran una correlación del $75 \%$ entre los $\mathrm{m}^{2}$ construidos totales y el PIB de las edificaciones, indicando que dicha variable refleja la dinámica de la actividad edificadora, los movimientos en cada segmento y la tendencia sectorial [10].

La figura 3 muestra la variación de ambas variables en cada período. La relación entre variables es comprobada por otros autores, quienes especifican que el crecimiento económico sectorial es el que genera el incremento en el área construida, y no en sentido contrario; [11].

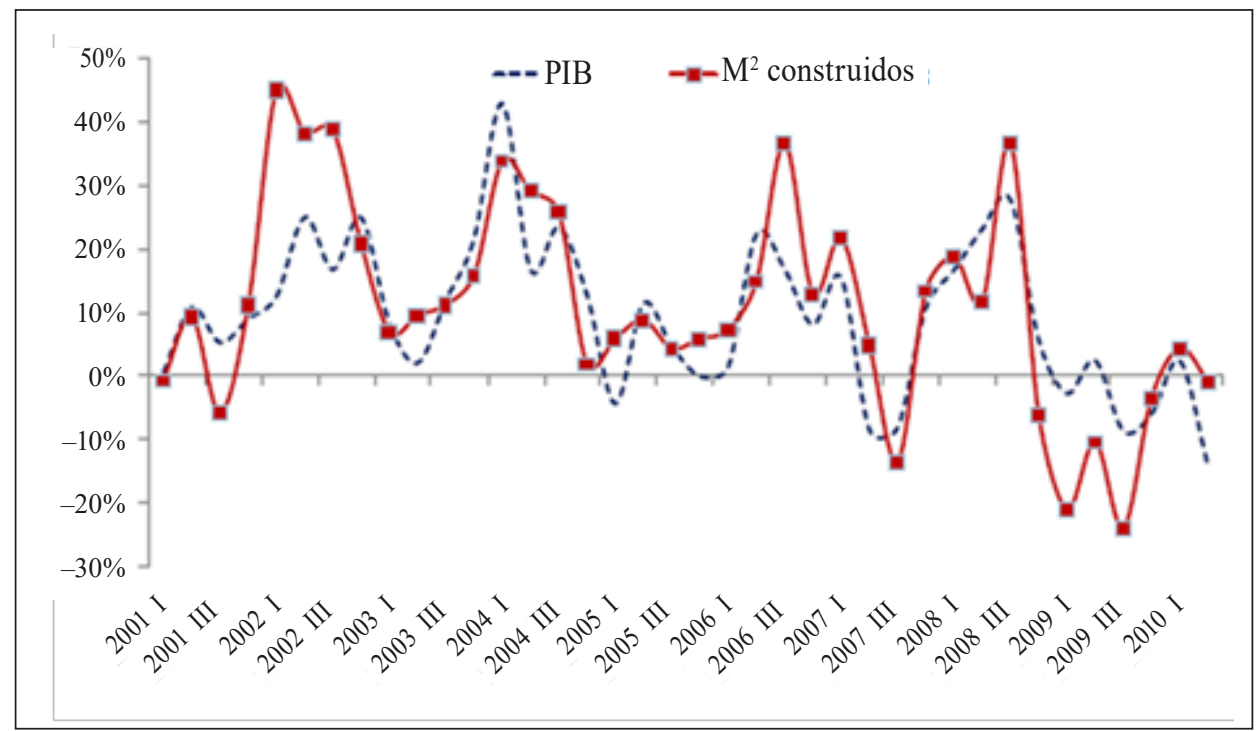

Figura 3. PIB de edificaciones frente a $\mathrm{m}^{2}$ construidos

Fuente: [10]

\subsection{Crédito hipotecario}

Cárdenas y Hernández [12] evalúan el efecto del crédito de vivienda en el ciclo constructivo, donde concluyen que el crédito hipotecario tiene un efecto positivo en la construcción. 
El crédito hipotecario que determina la demanda de vivienda nueva, representado en la tasa de interés del mismo, crea expectativa por ocupar un inmueble nuevo o usado minimizando la presión que el costo de la adquisición ejerce sobre el ingreso. El financiamiento de vivienda constituye opciones adecuadas en la capacidad de pago, $\mathrm{y}$ atrae la inversión de recursos [13].

\subsection{Déficit habitacional}

La vivienda debe satisfacer las necesidades de quien la habita; sirve para la defensa ante los rigores climáticos y para el desarrollo social y familiar; representa una inversión durable y transable [14]. Sus atributos se miden en función de los componentes básicos: estructura, servicios públicos, espacio, infraestructura de servicios comunales, localización y contorno. Las tres primeras categorías corresponden a la "casa" y las demás a su "entorno".

\subsection{Tasa de intervención}

La tasa de intervención -TIB- corresponde al elemento de control de flujo de capitales en el sistema económico del país, y se convierte en un mecanismo para evitar el "recalentamiento" de la economía en virtud de los resultados de crecimiento favorables, dado que permite minimizar la brecha de producto que mantiene la tasa de inflación cerca del objetivo del banco central. Con este criterio el Banco de la República modifica la tasa de interés mínima que cobra a las entidades financieras por los préstamos, o la tasa de interés máxima que paga por recibir dinero.

\subsection{Costos de construcción}

El índice de costos de construcción de vivienda-ICCV- es elaborado mensualmente por el DANE desde 1971, cuyo objetivo es medir la evolución del costo medio de la demanda de insumos para la construcción de vivienda, efectuado por medio de variaciones en precios de insumos al nivel nacional. El indicador se modificó en tres momentos: 1979, 1989 y 1999, y actualiza la base del análisis, incluye más zonas de cobertura y separa los tipos de construcción que conforman la muestra, de forma que pudiera brindar claridad de la calidad y estado de las obras.

\subsection{Precio de la vivienda}

El índice de precios de vivienda nueva-IPVN- representa el efecto de la oferta y la demanda de vivienda en el mercado nacional. El valor relativo de la vivienda en cada una de las ciudades se establece mediante el ajuste del indicador (tomando como base 100 el valor a diciembre de 2006) con el Índice del Precio al Consumidor 
total a junio de 2011, de acuerdo con la metodología de Clavijo et al. [7]. El resultado expresa la variación del precio de la vivienda en la última década para cada ciudad.

\subsection{Licencias de construcción}

Las licencias son reglamentadas en Colombia mediante el Decreto 2111 de 1997. El licenciamiento de construcción como proyección de la actividad futura sectorial es la variable empleada como aproximación para el fenómeno de construcción de vivienda, presentando una correlación del $97 \%$ con $l o s \mathrm{~m}^{2}$ construidos cuando se aplica un rezago de 12 meses [10].

\section{MODELO DINÁMICO DEL CICLO DE LA CONSTRUCCIÓN}

Analizando los determinantes con las variables presentadas anteriormente, se grafican cuatro diagramas causales individuales que representan ciclos de intervención. El primero es el ciclo básico de oferta y demanda; los restantes se asocian a los elementos que ocasionan alteración en la demanda de vivienda, y en consecuencia, bajan la participación de la construcción en el PIB nacional. Los elementos son: ciclo de costo, ciclo de oferta y ciclo de demanda.

\subsection{Diagramas causales}

\subsubsection{Ciclo básico de la construcción}

Para el modelado del ciclo constructivo se empleó la aplicación Powersim (en versión studio 2005). Se inició con la realización del diagrama causal básico, planteando las diferentes áreas en $\mathrm{m}^{2}$ entre cada uno de los niveles analizados en la figura 4, que corresponde al ciclo de realimentación negativo que representa el efecto entre la demanda y la oferta de vivienda.

Entre 2000 y 2009, el PIB per cápita aumentó en $28 \%$ en términos reales, el ingreso disponible aumentó en $30 \%$, y el subtotal de la cuenta de los hogares de este último indicador creció en $19 \%$ el mismo período. El segmento poblacional de 0-24 años, que constituye la demanda del mercado de vivienda aumentó un $3 \%$ en la población, pasando de $40 \%$ en el 2000 al $43 \%$ de 2010 . Finalmente, el tamaño promedio de los hogares en el país pasó de 4,2 personas en el 1997 por hogar a 3,7 en 2010 según Chirivi et al. [10].

Área mensual demandada. La demanda de vivienda está asociada a las mediciones de déficit habitacional establecido por el DANE, el cual involucra evaluación del censo habitacional con la proyección de nuevos hogares en formación que requerirán vivienda. Para el 2000, la construcción de viviendas en Colombia no alcanzaba 
25000 unidades anuales (2 083 viviendas/mes) como resultado de la crisis inmobiliaria de 1999 [15]. En el 2010 el Gobierno nacional fijó como meta de la construcción un millón de viviendas en el cuatrienio 2010-2014 [15].

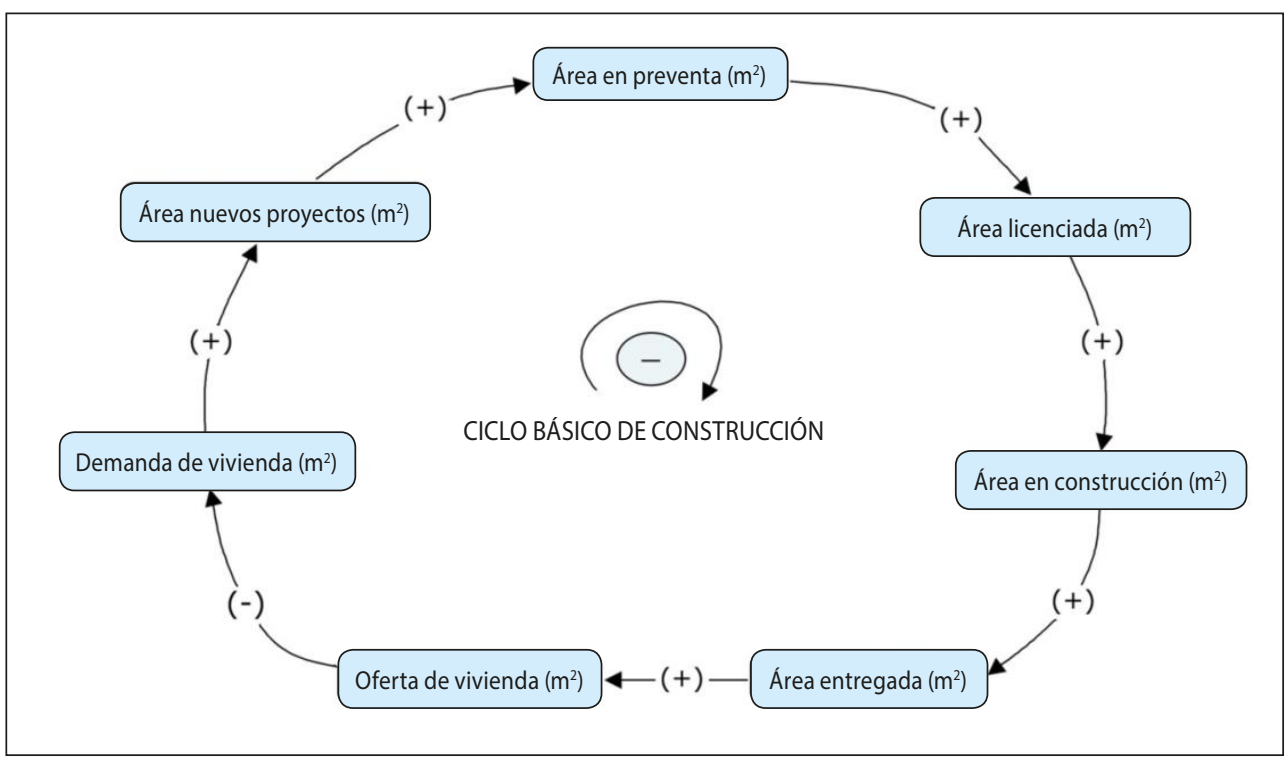

Figura 4. Ciclo básico de la construcción Fuente: elaboración propia

Área nuevos proyectos y área mensual en preventa. La viabilidad de un proyecto constructivo se prevé por parte de las promotoras de proyectos cuantificando los costos reales de construcción y las expectativas de venta, y estableciendo el margen de utilidad; las variaciones positivas en el precio de venta favorecen cuando se obtiene una rentabilidad mayor y las variaciones negativas afectan las utilidades reales del proyecto. No se considera para el modelo ningún caso en que el precio de venta esté por debajo del precio constructivo.

Área mensual licenciada. Los proyectos licenciados en las curadurías urbanas son aquellos en los cuales se detecta un interés específico de construcción por parte de promotoras de proyectos. La relación positiva entre el licenciamiento y el comportamiento del proceso constructivo es establecida por CAMACOL [10]. Se establece un período para el licenciamiento de los proyectos de vivienda cercano a los seis trimestres (18 meses), que coincide con las curvas de maduración que sirven de guía para determinar la incidencia del área en licencia en el PIB de edificaciones [10].

Área mensual en construcción. Los proyectos que inician construcción son aquellos que están financiados o con apalancamiento externo o una suficiente captación 
de recursos necesarios en el capital de trabajo. Los negocios cerrados en la etapa de preventa suponen una captación de dineros de "cuota inicial" por parte del constructor, que corresponde al porcentaje no cubierto mediante crédito.

Área terminada en oferta. Es la vivienda construida y lista para entregar a su propietario. La oferta de vivienda construida es el primer indicador que representa condiciones favorables o desfavorables de mercado. Los $\mathrm{m}^{2}$ construidos en vivienda nueva ponen a disposición de las familias una serie de activos adicionales que modifican la oferta y la demanda de vivienda.

\subsubsection{Ciclo de costos de construcción}

El aumento en los costos de construcción influye en el ciclo del modelo al nivel de utilidades, por lo cual no invierte en nuevos proyectos. Los costos pueden aumentar por la inflación o por la escasez de insumos para la construcción.

Este ciclo en la modelación requiere el manejo de un nivel en pesos, diferente al presentado anteriormente en $\mathrm{m}^{2}$.

En este caso la hipótesis a evaluar en el modelo se identificó por CAMACOL con un incremento de un $1 \%$ en los costos de construcción reduce en un 1,2 \% los $\mathrm{m}^{2}$ aprobados para la construcción de vivienda [16].

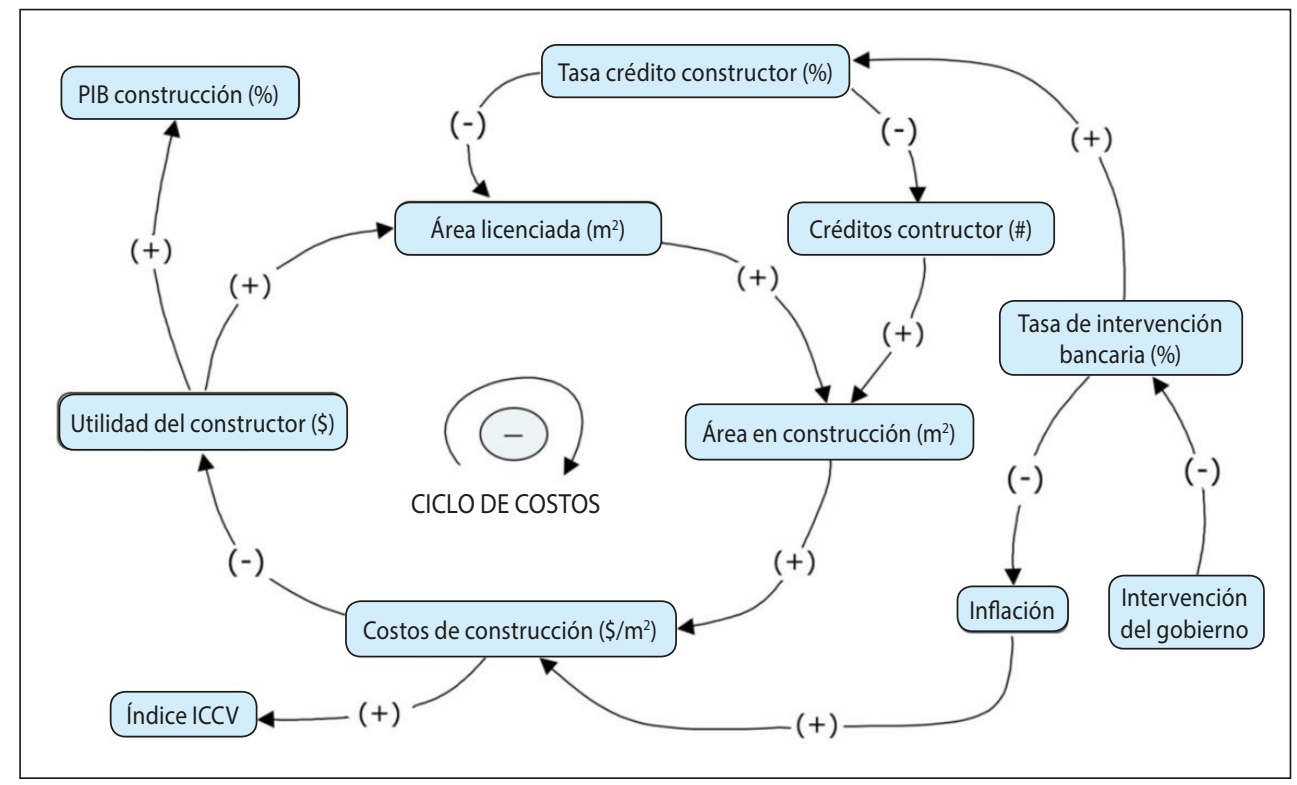

Figura 5. Ciclo de costos de construcción

Fuente: elaboración propia 
En la figura 5 se presenta el diagrama causal de este ciclo, planteando que el Gobierno influye en la tasa de intervención bancaria requerida para el control inflacionario. Sin embargo, mientras con la tasa controla el disponible que determina la inflación en la economía, aumenta el interés bancario a los créditos y minimiza el aporte del sector bancario a nuevos proyectos. Esta situación lleva a las promotoras a usar su capital como fuente de financiación y a adelantar una rigurosa selección de los mismos para reducir el riesgo.

Además de las variables analizadas en el ciclo anterior, se incluyen: costos unitarios de construcción, utilidad del constructor, tasa de intervención y de crédito constructor.

\subsubsection{Ciclo de la oferta de vivienda}

La figura 6 presenta los determinantes que actúan en el ciclo con el uso básico de las áreas que tiene cada una de las etapas del proceso constructivo y precio unitario de venta, determinante de la utilidad del constructor.

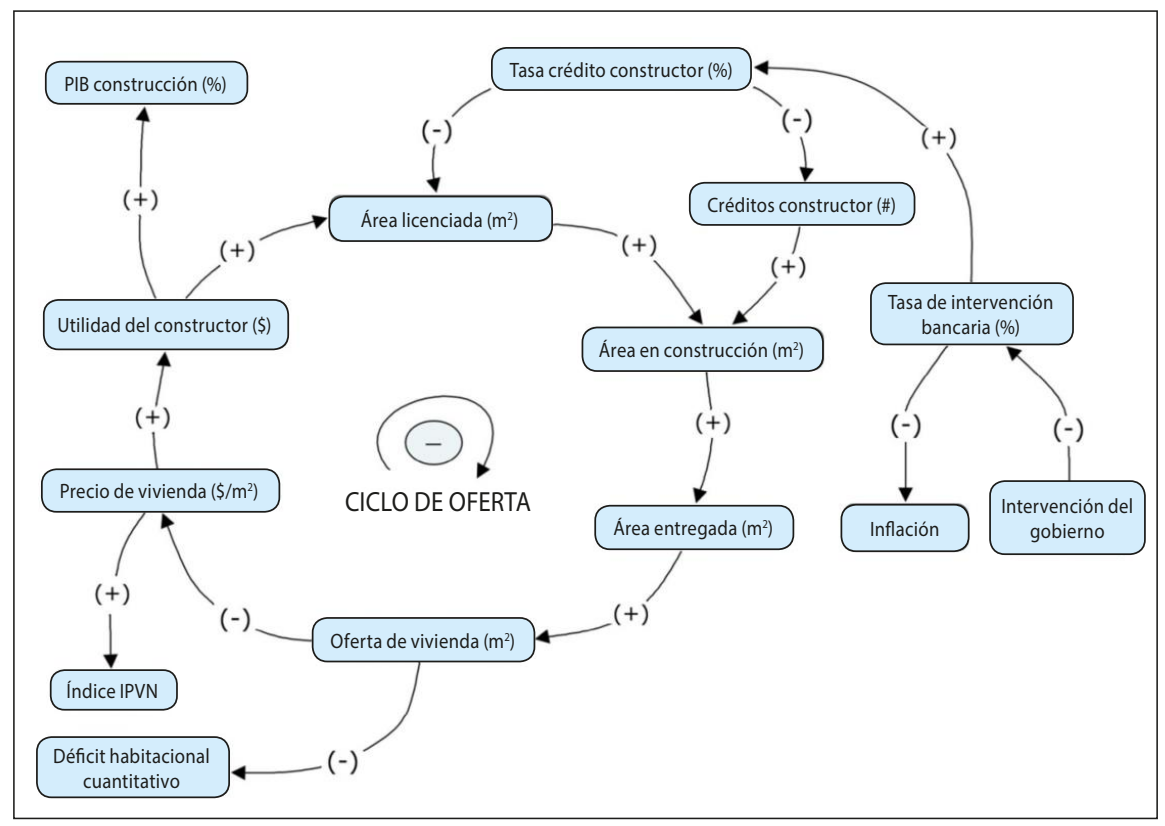

Figura 6. Ciclo de la oferta de vivienda

Fuente: elaboración propia

Se planea la participación del Gobierno en el ciclo de la oferta planteado mediante la modificación de la tasa de intervención bancaria -TIB-, que afecta el interés de crédito al constructor y obliga a invertir el capital.

Además de las variables analizadas en ciclos anteriores se incluye: 
Precio unitario de la vivienda: un incremento en el precio de venta de la vivienda es una condición que garantiza la restitución de los costos de construcción, más una rentabilidad superior a la mínima esperada.

\subsubsection{Ciclo de la demanda de vivienda}

En este numeral se presenta la metodología de análisis del Ciclo Básico de la Construcción mediante Dinámica de Sistemas, presentado en la figura 4, así como la metodología empleada para la validación del comportamiento cíclico del sector y las conclusiones obtenidas a partir del modelo.

Es el ciclo en el cual la política del Gobierno tiene mayor posibilidad de participación, desde la financiación de la tasa interbancaria y el establecimiento de demandas adicionales a las que se crean en la formación de nuevos hogares.

La demanda de vivienda nueva disminuye como resultado de la oferta creciente o aumenta como consecuencia de la depreciación de las tasas de créditos hipotecarios apalancadas vía subsidio. La figura 7 muestra los determinantes que afectan el proceso de demanda de vivienda.

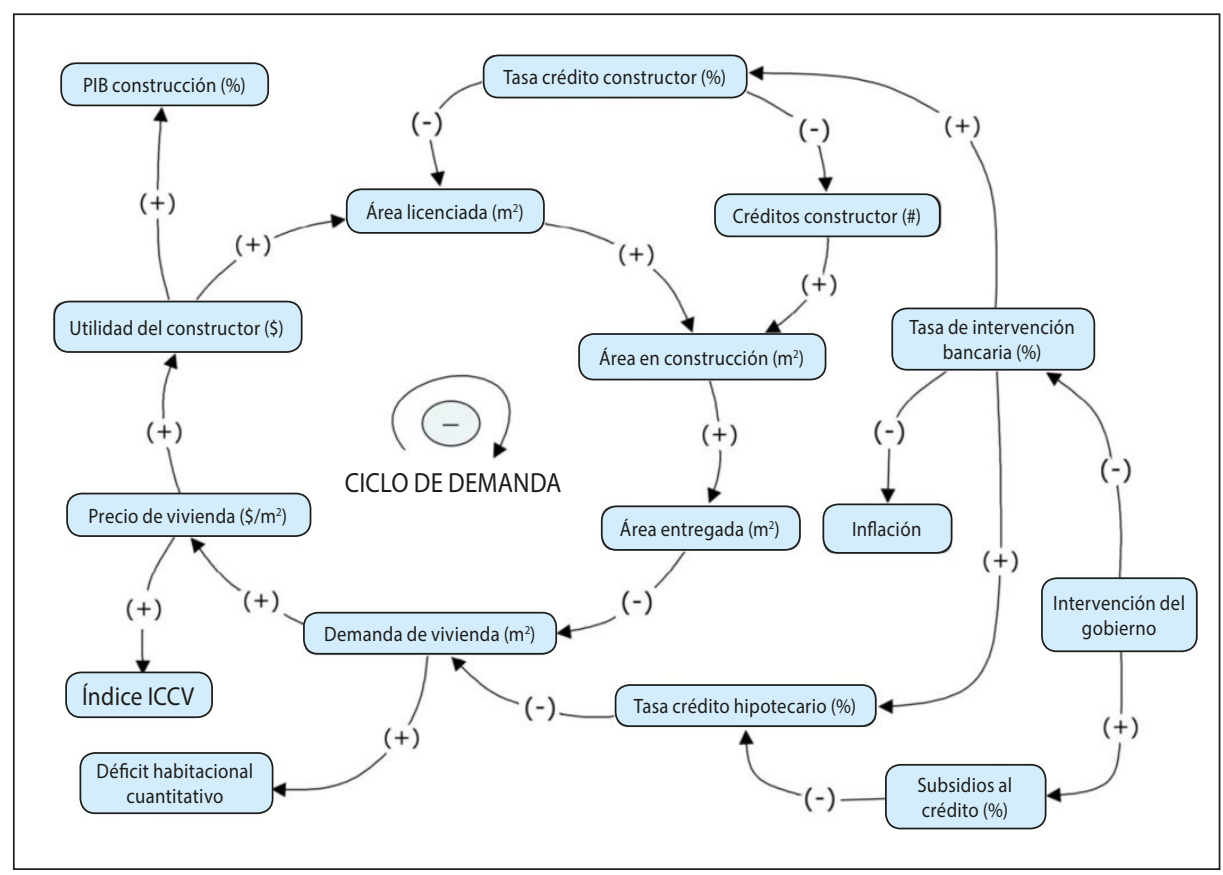

Figura 7. Ciclo de la demanda de vivienda Fuente: elaboración propia 


\subsection{RESULTADOSDE LA MODELACIÓN}

\subsubsection{Modelación del ciclo básico}

La modelación se hizo desde enero del 2000 a diciembre del 2014, para verificar las tendencias históricas sin modificaciones por parte del Gobierno. La figura 8 presenta el diagrama de flujos del ciclo básico, en que los niveles están dados en $\mathrm{m}^{2}$.

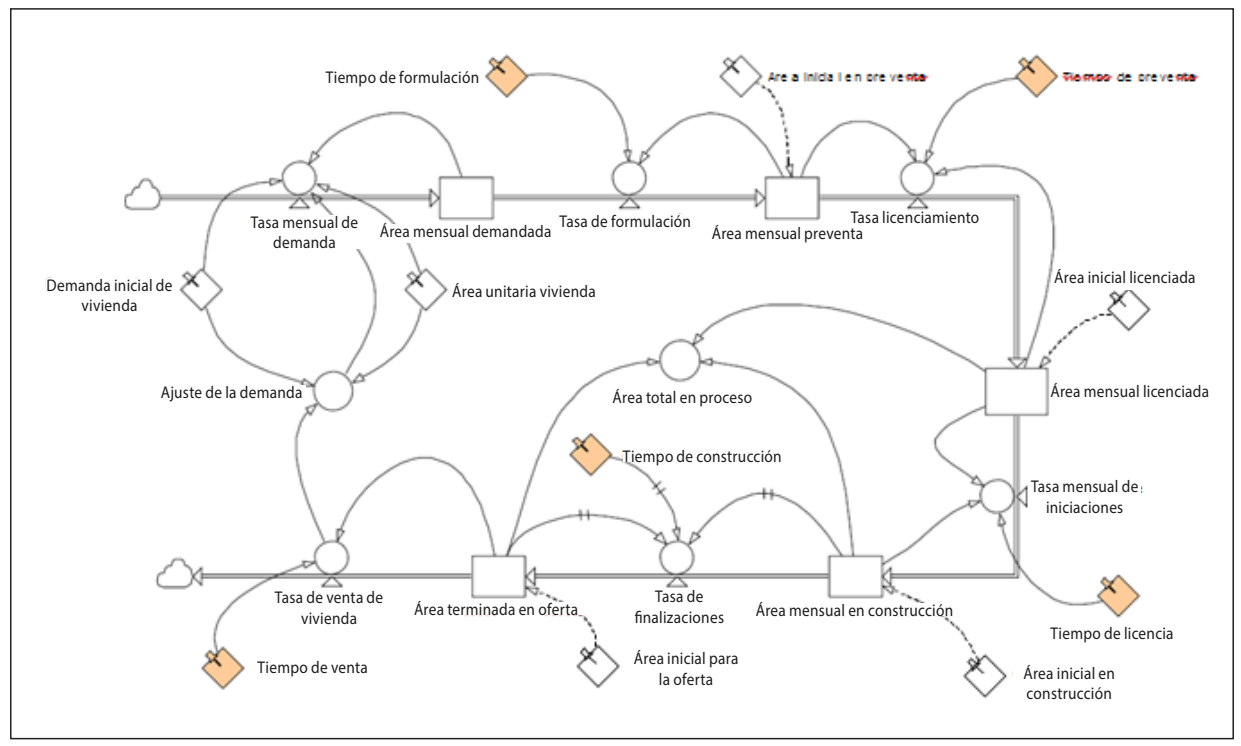

Figura 8. Diagrama de flujos del ciclo básico de la construcción

Fuente: elaboración propia

Generalmente, los tiempos de construcción son menores a los empleados en la preventa y dependen de los niveles de riesgo asumidos por las constructoras; ellas emplean en promedio de 12 a 24 meses para gestionar la instalación de sala de ventas, evaluación de mercado, diseños de detalle y licenciamiento.

Se emplea una demanda inicial de 8000 viviendas mensuales (promedio mensual entre 2000-2010), área de $60 \mathrm{~m}^{2}$ por unidad, correspondiente a la VIS.

Los niveles inician en ceros para evaluar el comportamiento cíclico y los tiempos de demora que determinan los flujos se resaltan en el módulo de programación.

La modelación muestra en la figura 9 el comportamiento cíclico de la construcción con períodos completos de desaceleración y aceleración de 90 meses, es decir, 7,5 años para la demanda; 86 meses, es decir, 7,2 años para la preventa y 82 meses, es decir, 6,8 años para el licenciamiento, construcción y oferta de vivienda. CAMACOL, en el año 2009, identificó un ciclo de 6 años promedio para Colombia [1], indicando que el modelo, tiene un comportamiento similar a la realidad. 
El modelo muestra un máximo nivel de área licenciada en el mes 35 (mes 3 en la figura 9), equivalente al mes de noviembre de 2002, con $11679543 \mathrm{~m}^{2}$, mientras que el máximo de área en construcción es en el mes 44, equivalente a agosto de 2003, indicando un rezago de nueve meses entre el pico de licenciamiento y el pico de construcción. Para CAMACOL, la realidad del rezago es de doce meses.

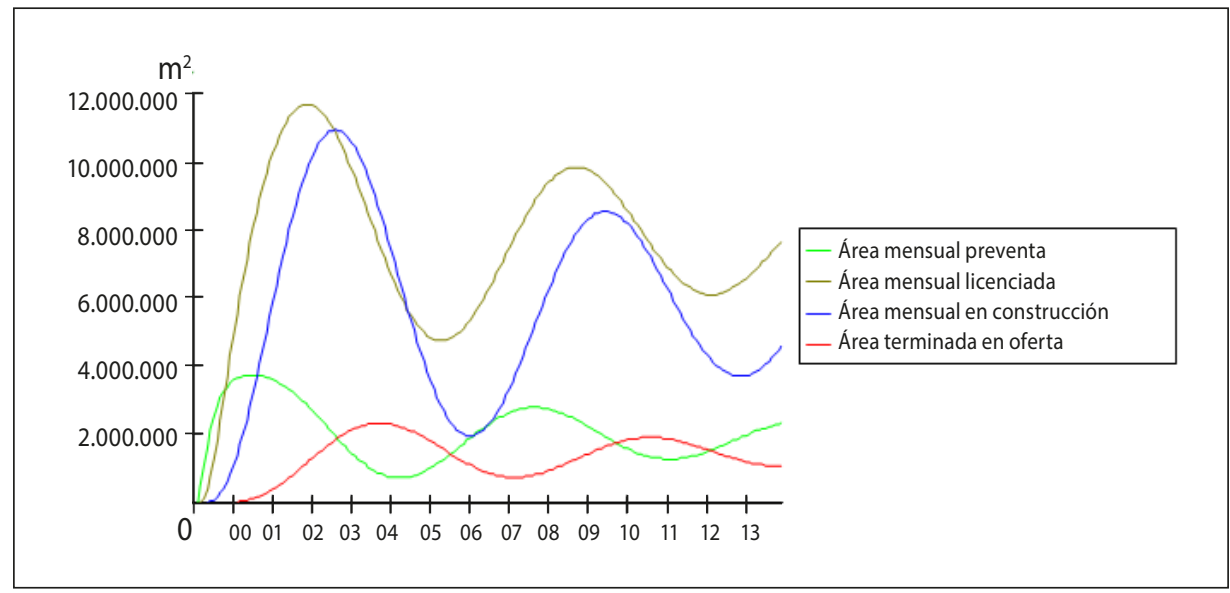

Figura 9. Resultados de la modelación inicial Fuente: elaboración propia

\subsubsection{Validaciones del modelo del ciclo básico}

\section{Variación en los tiempos de actividades}

Ajustar los tiempos de las distintas actividades relacionadas en el ciclo básico tiene como objetivo lograr el promedio de 6 años como ciclo para Colombia [1]. Se evalúa la sensibilidad de los tiempos de licenciamiento y construcción como actividades más representativas a partir de la modelación básica de demanda promedio presentada en la figura 9. Para evaluar la incidencia se modelan variaciones del $25 \%$ en el tiempo (12 y 20 meses, respectivamente). La modificación del tiempo de licenciamiento a 12 meses observa una disminución del $20 \%$ en los niveles alcanzados por la actividad a $9352191 \mathrm{~m}^{2}$, y reducción del 6,1 \% (77 meses o 6,4 años). Los resultados se muestran en la figura 10 .

Se concluye que el efecto representativo de la modificación en tiempos de licenciamiento se da en $\mathrm{m}^{2}$, y no en tiempos del ciclo.

Evaluando la incidencia, se modelaron variaciones del $25 \%$ en el tiempo inicial (9 y 15 meses). Se observa disminución del $28,6 \%$ en los niveles alcanzados, a 7 $817001 \mathrm{~m}^{2}$, y una reducción en el ciclo de 5,8 \% (69 meses o 5,8 años), cerca al ciclo promedio. En la figura 11 se observa el tiempo de construcción a 9 meses. 


\section{Variaciones en la demanda}

El análisis de sensibilidad tiende a ajustar las condiciones esperadas para validar con los resultados del mercado. A partir de la demanda de vivienda como elemento dinamizador del mercado se efectúan modelaciones con 4 000, 6000,10000 y 12000 viviendas/mes de demanda promedio y constante, sin modificar ningún otro criterio de tiempo. En la figura 12 se muestra la demanda de las 4000 viviendas.

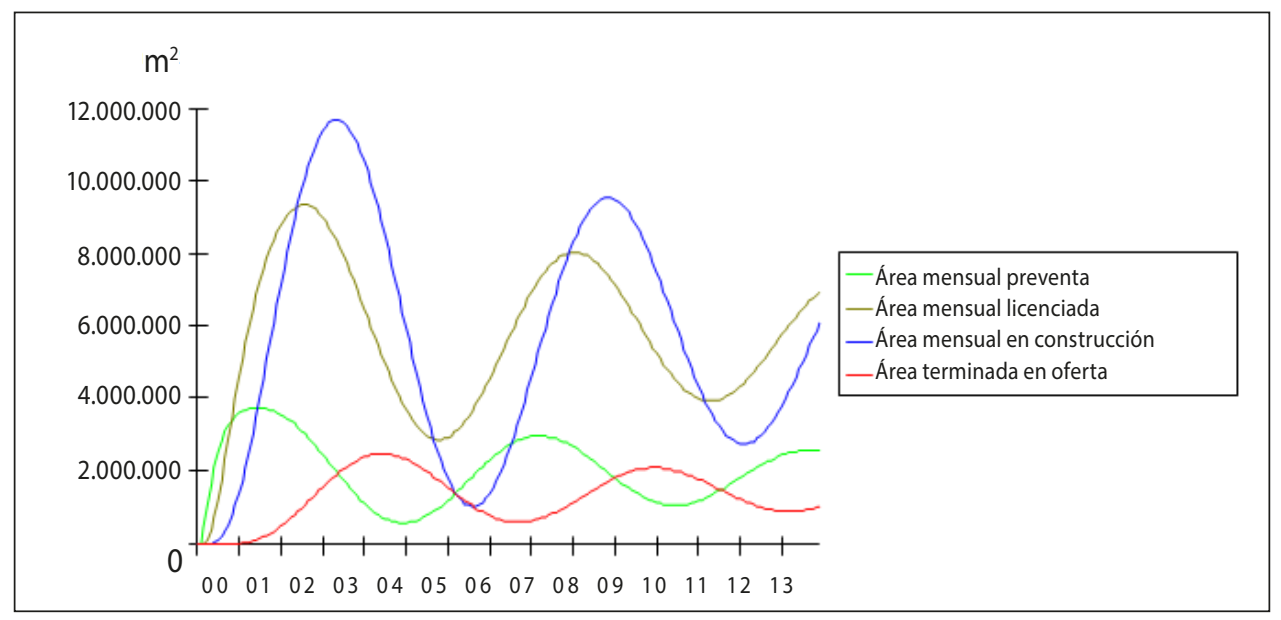

Figura 10. Tiempo de licenciamiento a 12 meses

Fuente: elaboración propia

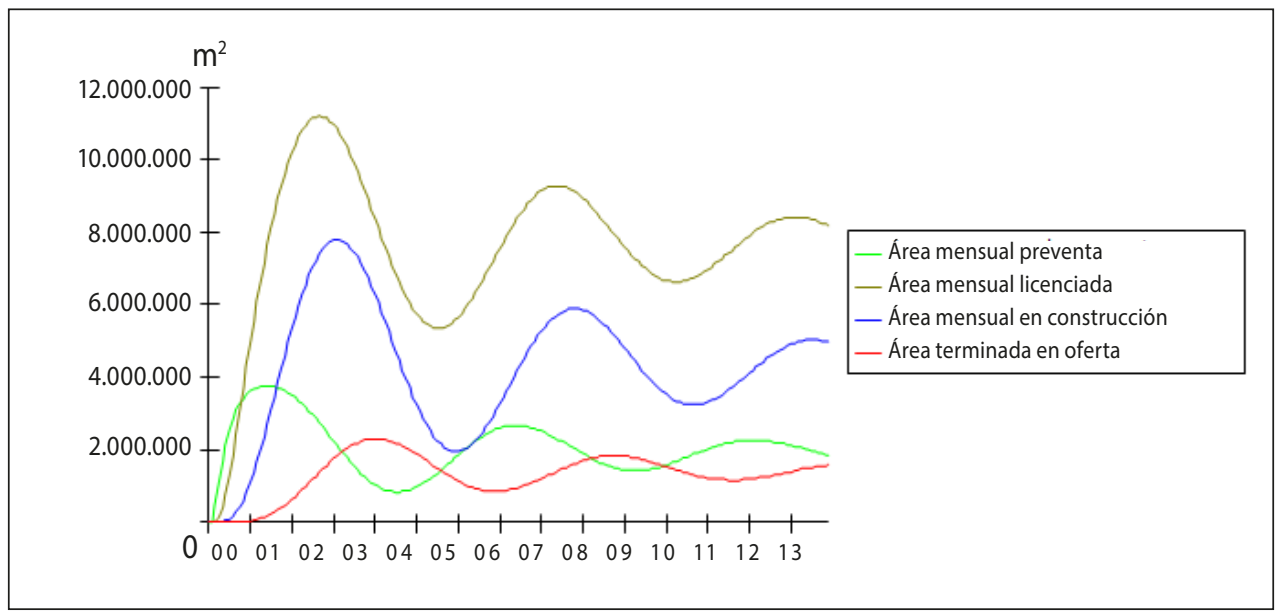

Figura 11. Tiempo de construcción de 9 meses

Fuente: elaboración propia

Se modeló con demanda de 8000 viviendas/mes hasta el mes 90 y 16000 viviendas/mes a partir del mes 91 de la modelación. Ver figura 13. 


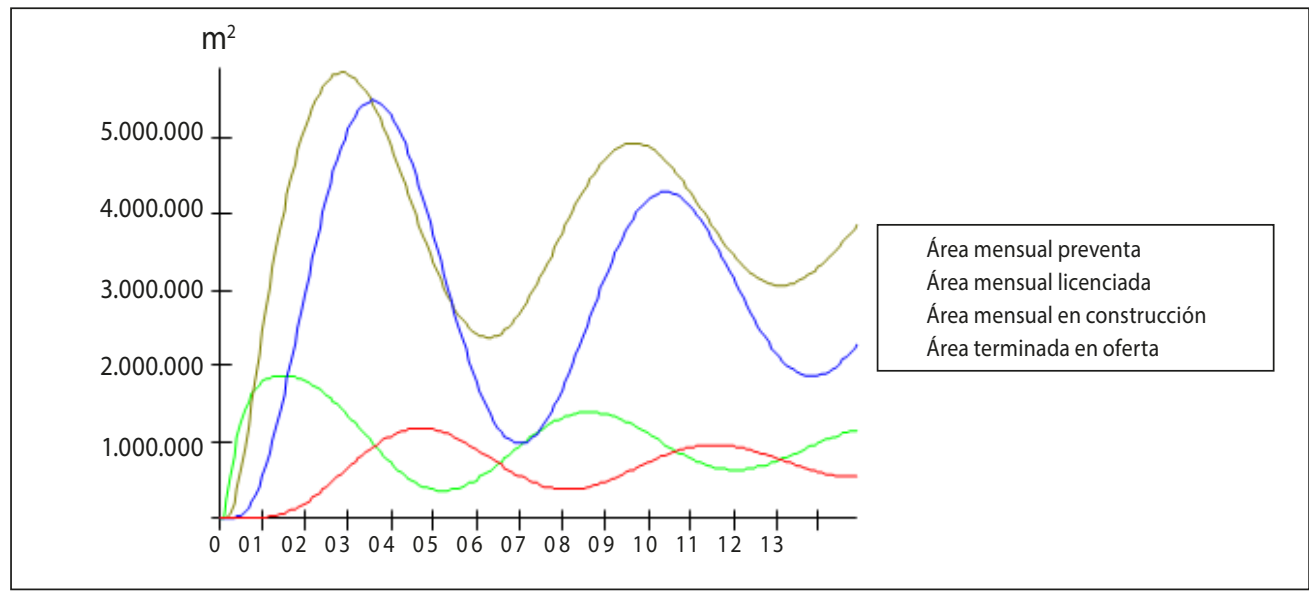

Figura 12. Demanda constante de 4000 viviendas por mes Fuente: elaboración propia

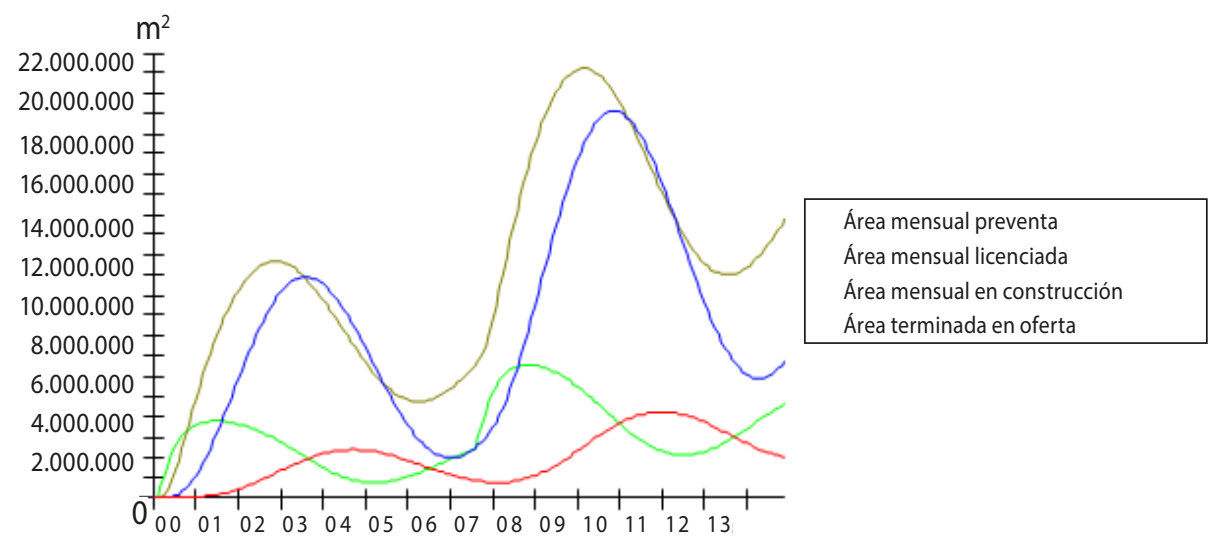

Figura 13. Ciclo con duplicación de la demanda en el mes 90 Fuente: elaboración propia

El incremento en la demanda afecta el ciclo, de 82 a 87 meses (7,3 años), pero incrementó más el licenciamiento, $42 \%$, que la construcción, $25 \%$, lo que corresponde a los tiempos en que la modificación aplica a cada actividad.

La aplicación de las políticas de vivienda del Gobierno nacional en el denominado "gran salto" para el periodo 2010-2014 supone la construcción de 1000000 de viviendas adicionales, que en términos prácticos corresponde a 250000 viviendas al año y cerca de 21000 viviendas al mes. La política pretende generar un efecto anticíclico en el histórico de la actividad. La variación de la demanda planteada por el 
Gobierno se modeló con demanda constante de 8000 viviendas por mes y un incremento a 21.000 viviendas por mes hasta el 2014, incremento promedio del $162 \%$.

Finalmente, en el segundo trimestre del año 2015 el subsector de las edificaciones y obras civiles tuvo aumentos de $9.1 \%$ y $8.4 \%$, que lleva al crecimiento del valor agregado del sector construcción de $8.7 \%$ [18].

\section{CONCLUSIONES}

El mercado de la construcción es influenciado por el Gobierno, es decir, altera la demanda de vivienda de acuerdo con las normas y políticas del momento.

La oferta y la demanda son determinantes en la construcción de vivienda y están sujetas a variables como los subsidios, la tasa de crédito hipotecario, la cual depende de la tasa de usura que fije el Banco de la República, planes de inversión de constructores privados y normas asociadas a las licencias; estas variables fueron modeladas mediante dinámica de sistemas.

El ciclo de la construcción modelado en retardos y efectos de cada uno de los indicadores se configuró para representar los elementos históricos y evaluar las incidencias de cambios propuestos al futuro.

Los ciclos planteados a partir de los determinantes estudiados obedecen a procesos dinámicos, de acuerdo con el análisis independiente en diagramas causales, pero la transformación a diagramas de flujos requiere herramientas de dinámica de sistemas para la interpretación completa.

La implementación de niveles relacionados con los ingresos de empresas constructoras, los costos unitarios de construcción y precios unitarios de venta permitieron modelar la utilidad teórica sectorial, la cual podrá ser contrastada con los resultados del PIB.

Los tiempos de las actividades empleadas en cada una de las etapas tienen influencia en el resultado de acumulación y gestión de la actividad edificadora que en la definición del ciclo dinámico. Es necesario evaluar efectos conjuntos de modificación de tiempos y demandas variables para ajustar políticas a mediano y largo plazo [17].

La modificación de la demanda de vivienda como medida anti-cíclica para incrementar la participación del sector constructivo de edificaciones es más efectiva en el licenciamiento que en la construcción.

El mayor valor generado en el ejercicio anual se da por los incrementos en la actividad edificadora, influenciado por la demanda más que por los incrementos en las 
utilidades netas de proceso.

La política "gran salto", propuesta por el Gobierno como medida anti-cíclica en la construcción, determina un nuevo panorama para el ciclo constructivo que, de no modificar las condiciones planteadas, presentará un incremento mínimo en el área licenciada a finales del año 2015 y en el área construida a finales del 2016.

\section{REFERENCIAS}

[1] A. Cárdenas et al., "Ciclos de la actividad edificadora en el mundo y en Colombia", Informe

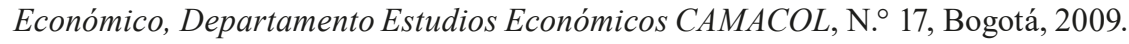

[2] E. Saldarriaga, Determinantes del sector de la construcción en Colombia. Bogotá, 2006.

[3] A. W. Throop, "Financial deregulation, interest rates, and the housing cycle", Federal Reserve Bank of San Francisco Economic Review, pp. 73-68, 1986.

[4] R. H. Topel, y S. Rosen, "Housing Investment in the United States", Journal of Political Economy, vol. 96, N. 4, pp. 718-740, 1988.

[5] M. Cárdenas, y R. Bernal, "Auge y Crisis de la Construcción en Colombia: Causas y Consecuencias”, Revista CAMACOL, vol. 21, N. ${ }^{\circ} 1,1997$.

[6] M. Cárdenas et al., "Determinantes de la Actividad Constructora en Colombia," Estudio realizado por Fedesarrollo para la Constructora Colpatria, Bogotá, 2004.

[7] S. Clavijo et al., La vivienda en Colombia: sus determinantes socio-económicos y financieros. Bogotá, Colombia: $\quad$ Banco de la República, Borradores de Economía, N. 300, 2004.

[8] CAMACOL, El sector de la construcción en Colombia: hechos estilizados y principales determinantes del nivel de actividad. Bogotá: Departamento de Estudios Económicos de CAMACOL, 2008.

[9] E. Chirivi, y L. D. Pulido, Lecciones del Congreso Colombiano de la Construcción 2009: Competitividad para construir el futuro. Bogotá: Departamento Estudios Económicos CAMACOL, Informe Económico N. 19, 2009.

[10] E. Chirivi et al., Entendiendo el PIB de edificaciones. Bogotá: Departamento Estudios Económicos CAMACOL, Informe Económico No. 26, 2011.

[11] M. Villegas, y L. M. Armas, "La construcción de vivienda en Colombia, el PIB y otros factores; un estudio de ciclos y tendencias" Universidad ICESI, Facultad de Ciencias Económicas y Administrativas, 2010.

[12] M. Cárdenas, y M. Hernández, El sector financiero y la vivienda. Estudio realizado por Fedesarrollo para Asobancaria, Bogotá, 2006. 
[13] G. González, El crédito hipotecario y el acceso a la vivienda para los hogares de menores ingresos en América Latina. Santiago de Chile: Naciones Unidas, CEPAL, 2002, 105 pp.

[14] DANE. Metodología déficit de vivienda. Actualización 2009, N. 79. Bogotá, Colombia: Colección Documentos, DANE, 2009, 56 pp.

[15] B. Chirivi et al., "La vivienda 2011-2014: "El Gran Salto" en la producción habitacional," Departamento Estudios Económicos CAMACOL, Informe Económico N. ${ }^{\circ}$ 23, 2010.

[16] A. Cárdenas, y G. P. Castellanos, “ Determinantes del ciclo de la construcción en Colombia,” Departamento Estudios Económicos CAMACOL, Informe Económico No. 11, 2008.

[17] P. Quiroz et al., "Los precios de la vivienda desde su contexto, medición y algunos determinantes," Departamento Estudios Económicos CAMACOL, Informe Económico N. 45, 2012.

[18] DANE, «Indicadores Economicos Alrededor de la Construcción-II trimestre de 2015,»09 octubre 2015. [En línea]. Available: http://www.dane.gov.co/files/investigaciones/boletines/ pib_const/Bol_ieac_IItrim15.pdf. [Último acceso: 29 octubre 2015]. 
